\title{
A Comparison Study of Two Fuzzy-based Handover Systems for Avoiding Ping-Pong Effect in Wireless Cellular Networks
}

\author{
Gjergji Mino $^{\dagger}$, Leonard Barolli ${ }^{\ddagger}$, Arjan Durresi ${ }^{\dagger \dagger}$, Fatos Xhafa ${ }^{\dagger \ddagger}$, Akio Koyama ${ }^{\ddagger \ddagger}$ \\ $\lceil$ Graduate School of Engineering \\ Fukuoka Institute of Technology (FIT) \\ 3-30-1 Wajiro-Higashi, Higashi-Ku, Fukuoka 811-0295, Japan \\ E-mail: gjmino@gmail.com \\ ${ }^{\ddagger}$ Department of Information and Communication Engineering \\ Fukuoka Institute of Technology (FIT) \\ 3-30-1 Wajiro-Higashi, Higashi-ku, Fukuoka 811-0295, Japan \\ E-mail: barolli@fit.ac.jp \\ $\dagger \dagger$ Department of Computer and Information Science \\ Indiana University Purdue University at Indianapolis (IUPUI) \\ 723 W. Michigan Street SL 280, Indianapolis, IN 46202, USA \\ E-mail: durresi@cs.iupui.edu \\ $\dagger$ Department of Languages and Informatics Systems \\ Technicnical University of Catalonia \\ Jordi Girona 1-3, 08034 Barcelona, Spain \\ E-mail: fatos@1si.upc.edu \\ ${ }^{\ddagger}$ Department of Informatics, Yamagata University \\ 4-3-16 Jonan, Yonezawa 992-8510, Yamagata, Japan \\ E-mail: akoyama@yz.yamagata-u.ac.jp
}

\begin{abstract}
Wireless mobile networks and devices are becoming increasingly popular to provide users the access anytime and anywhere. The mobile systems are based on cellular approach and the area is covered by cells that overlap each other. Many handover algorithms are proposed in the literature. However, to make a better handover and keep the QoS in wireless networks is very difficult task. For this reason, new intelligent algorithms should be implemented to deal with this problem. In this paper, we carried out a comparison study of two handover systems based on fuzzy logic. We implement two Fuzzy-Based Handover Systems (FBHS). We call them FBHS1 and FBHS2. The performance evaluation via simulations shows that FBHS2 has better behavior than FBHS2 and can avoid ping-pong effect in all simulation cases.
\end{abstract}

\section{Introduction}

During the last few years wireless multimedia networks have been a very active research area [1,2]. The QoS support for future wireless networks is a very important prob- lem. To guarantee the QoS, a good handover strategy is needed in order to balance the call blocking and call dropping for providing the required QoS $[3,4]$. In the future, the wireless networks will adopt a micro/pico cellular architecture. However, smaller cell size naturally increases the number of handoffs a Mobile Station (MS) is expected to make $[5,6]$.

Many metrics have been used to support handover decisions, including Received Signal Strength (RSS), Signal to Interference Ratio (SIR), distance between the mobile and BS, traffic load, and mobile velocity, where RSS is the most commonly used one. The conventional handover decision compares the RSS from the serving BS with that from one of the target BSs, using a constant handover threshold value (handover margin). However, the fluctuations of signal strength associated with shadow fading cause the pingpong effect [7].

Many investigations have addressed handover algorithms for cellular communication systems. However, it is essentially complex to make handover decision considering multiple criteria. Sometimes, the trade-off of some criteria should be considered. Therefore, heuristic approaches 
based on Neural Networks (NN), Genetic Algorithms (GA) and Fuzzy Logic (FL) can prove to be efficient for wireless networks $[8,9,10,11,12,13]$. In [10], a multi-criteria handover algorithm for next generation tactical communication systems is introduced. The handover metrics are: RSS from current and candidate base transceivers, ratio of used soft capacity to the total soft capacity of base transceivers, the relative directions and speeds of the base transceivers and the mobile node. In [11], a handover algorithm is proposed to support vertical handover between heterogeneous networks. This is achieved by incorporating the mobile IP principles in combination with FL concepts utilizing different handover parameters. In [12,13], we proposed and implemented a Fuzzy-Based Handover System (FBHS). We showed that the proposed system had a good behavior for handover enforcement, but in some cases could not avoid the ping-pong effect.

In this paper, we carry out a comparison study of two FBHS: FBHS1 and FBHS2. The performance evaluation via simulations shows that new implemented FBHS2 has better behavior than FBHS2 and can avoid ping-pong effect in all simulation cases.

The structure of this paper is as follows. In Section 2, we present the handover decision problem. In Section 3, we give a brief introduction of RW model. In Section 4, we introduce the implemented FNHS. In Section 5, we discuss the simulation results. Finally, some conclusions are given in Section 6.

\section{Handover Decision Problem}

Handoffs which are consistently both accurate and timely can result in higher capacity and better overall link quality than what is available with today systems $[14,15]$. Now with increasing demands for more system capacity, there is a trend toward smaller cells, also known as microcells. Handoffs are more critical in systems with smaller cells, because for a given average user speed, handoff rates tend to be inversely proportional to cell size [5].

The main objectives of handover are link quality maintenance, interference reduction and keeping the number of handoffs low. Also, a handover algorithm should initiate a handoff if and only if the handoff is necessary. The accuracy of a handover algorithm is based on how the algorithm initiates the handover process. The timing of the handoff initiation is also important. There can be deleterious effects on link quality and interference if the initiation is too early or too late. A timely handover algorithm is one which initiates handoffs neither too early nor too late.

Because of large-scale and small-scale fades are frequently encountered in mobile environment, it is very difficult for handover algorithm to make an accurate and timely decision. Handover algorithms operating in real time have to make decisions without the luxury of repeated uncorre-

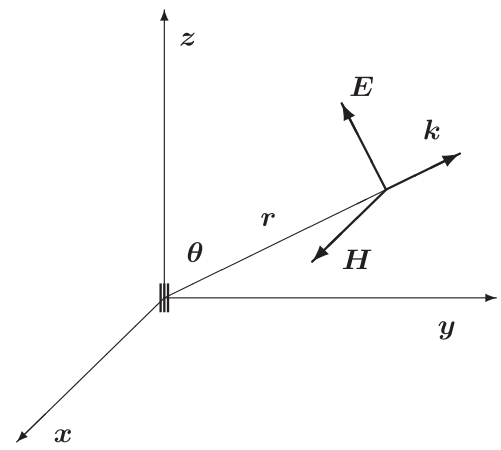

Figure 1. Dipole antenna.

lated measurements or the future signal strength information. It should be noted that some of handover criteria information can be inherently imprecise, or the precise information is difficult to obtain. For this reason, we propose a FL-based approach, which can operate with imprecision data and can model nonlinear functions with arbitrary complexity.

\section{RW Model}

The Monte Carlo (MC) method is a technique that uses random numbers and probability to solve problems. It is often used when the model is complex, nonlinear, or involves more than just a couple uncertain parameters.

The MC method can be used for analyzing uncertainty propagation, where the goal is to determine how random variation, lack of knowledge, or error affects the sensitivity, performance, or reliability of the system that is being modeled. MC simulation is categorized as a sampling method because the inputs are randomly generated from probability distributions to simulate the process of sampling from an actual population. The data generated from the simulation can be represented as probability distributions (or histograms) or converted to error bars, reliability predictions, tolerance zones, and confidence intervals.

We use the MC method for realizing RW model. We consider a 2-dimensional field. The initial position is considered as a origin point and we decided based on MC method the moving pattern for each walk. If we consider $n$ user movements and the angle $\theta$ and distance $d$ for each walk are generated by general or Gaussian distribution, when the movement changes in $x$ and $y$ directions are $\Delta x$ and $\Delta y$, respectively, then we have the following relations.

$$
\begin{gathered}
\Delta x_{n}=d_{n} \cos \theta_{n}, \quad \Delta y_{n}=d_{n} \sin \theta_{n} \\
x_{n+1}=x_{n}+\Delta x_{n}, \quad y_{n+1}=y_{n}+\Delta y_{n}
\end{gathered}
$$

The Base Station (BS) position can be expressed by Cartesian coordinates. By converting Cartesian coordinates to polar ones, we can calculate the angle $\theta$.

We consider that in the cellular system each cell has a hexagonal shape and the BS is located in the center of the 


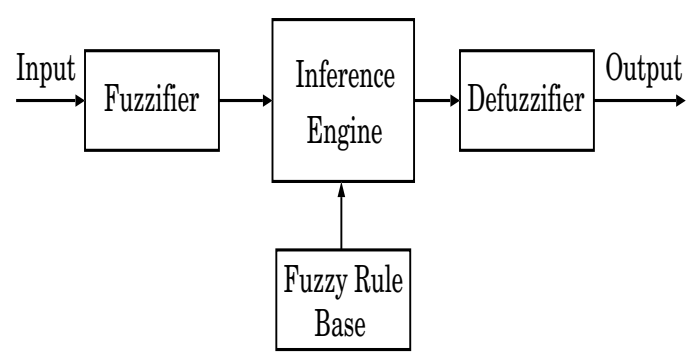

Figure 2. FLC structure.
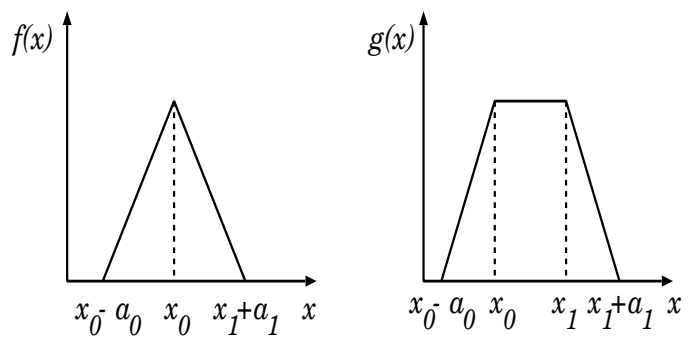

Figure 3. Membership function shapes.

cell. The angle $\theta$ between Dipole Antenna (DA) and vector $\boldsymbol{r}$ is $D(\theta)=\sin \theta$. If we consider the transmission power as $W$, the antenna radiation intensity can be calculated as follows:

$$
\boldsymbol{E}=\sqrt{45 W} \sin \theta \frac{e^{-j \kappa r}}{r^{n}} \boldsymbol{u}_{\mathbf{0}}
$$

where, the DA gain is $G=1.5$ and $\boldsymbol{u}_{0}$ is the unit vector that shows DA direction. In Fig. $1 \boldsymbol{u}_{0}$ is in $Z$ direction.

In Eq.(3), when $\theta=90^{\circ}$, the $E$ value will be maximal in horizontal direction. However, in real situations, the direction of antenna is not set $90^{\circ}$ in order to cover better the cell area. If we consider the beam tilting angle and the distance, the $E$ can be calculated by the following equation.

$$
\boldsymbol{E}=\sqrt{45 W} \sin (\theta-\phi) \frac{e^{-j \kappa r}}{r^{n}} \boldsymbol{u}_{\mathbf{0}}
$$

\section{Implemented System Models}

\subsection{FLC Structure}

The Fuzzy Logic Controller (FLC) is the main part of the FBHS and its basic elements are shown in Fig. 2. They are the fuzzifier, inference engine, Fuzzy Rule Base (FRB) and defuzzifier. As shown in Fig. 3, as membership functions we use triangular and trapezoidal membership functions because they are suitable for real-time operation [16].

In Fig. 3, $x_{0}$ in $f($.$) is the center of triangular function,$ $x_{0}\left(x_{1}\right)$ in $g($.$) is the left (right) edge of trapezoidal func-$ tion, and $a_{0}\left(a_{1}\right)$ is the left (right) width of the triangular or trapezoidal function.

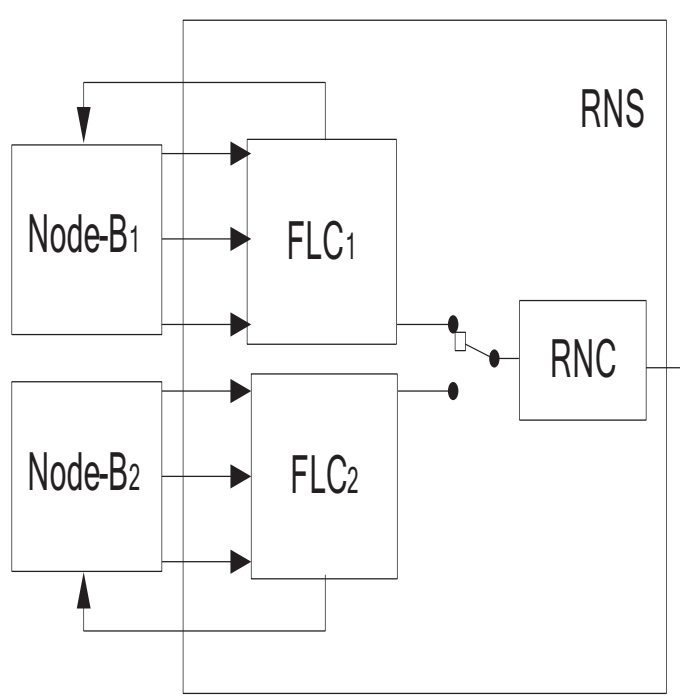

Figure 4. FBHS1 model.
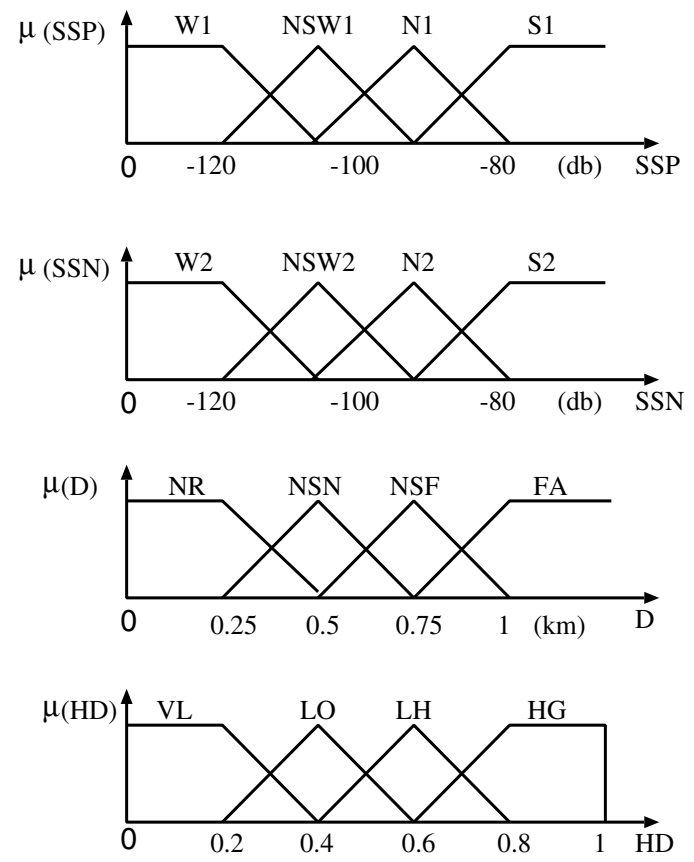

Figure 5. FBHS1 membership functions.

\subsection{Design of FBHS1}

The FBHS1 model is shown in Fig. 4. The Node_B shows the wireless transmitter and receiver of BS and RNS indicates Radio Network System.

As the input linguistic parameters for FBHS1, we consider: Signal Strength from the Present BS (SSP), Signal Strength from the Neighbor BS $(S S N)$, and the distance of MS from BS $(D)$. The output linguistic parameter is Handover Decision $(H D)$.

The term sets of $S S P, S S N$ and $D$ are defined respectively as: 
Table 1. FRB1.

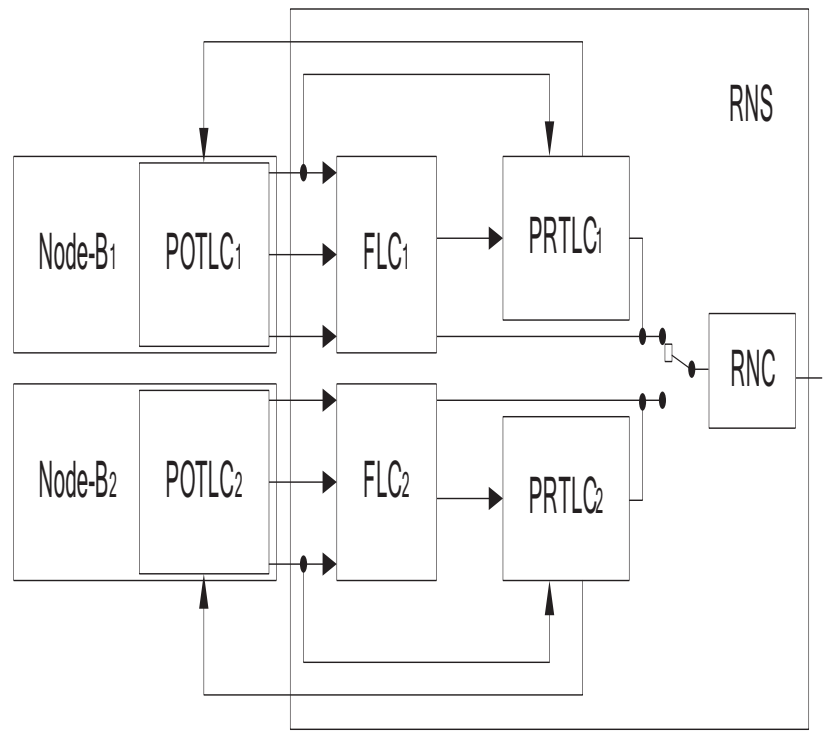

Figure 6. FBHS2 model.

$$
\begin{aligned}
T(S S P) & =\{\text { Weak, Not So Weak, Normal, Strong }\} \\
& =\{\text { W1, NSW1,N1,S1\}; } \\
T(S S N) & =\{\text { Weak, Not So Weak, Normal, Strong }\} \\
& =\{\text { W2, NSW } 2, N 2, \text { S } 2\} ; \\
T(D) & =\{\text { Near, Not So Near, Not So Far, Far }\} \\
& =\{\text { NR,NSN,NSF,FA } .
\end{aligned}
$$

The output linguistic parameter $T(H D)$ is defined as $\{$ Very Low, Low, Little High, High $\}=$ $\{V L, L O, L H, H G\}$.

The membership functions of FBHS1 are shown in Fig. 5. The FRB1 forms a fuzzy set of dimensions $|T(S S P)| \times$ $|T(S S N)| \times|T(D)|$, where $|T(x)|$ is the number of terms on $T(x)$. The FRB1 is shown in Table 1 and has 64 rules. The control rules have the following form: IF "conditions" THEN "control action".

\subsection{Design of FBHS2}

The FBHS2 model is shown in Fig. 6. In this system, the same as FBHS1 model, the $N_{\text {ode }} B$ shows the wireless transmitter and receiver of BS, RNS indicates Radio Network System. While, the POTLC stands for Post Test-Loop Controller and PRTLC for Pre Test-Loop Controller.

Different from FBHS1, in FBHS2 we consider as the input parameter the Change of the Signal Strength of Present BS (CSSP). While two other parameters: Signal Strength from the Neighbor BS $(S S N)$, and the distance of MS from

\begin{tabular}{|c|c|c|c|c|}
\hline Rules & SSP & SSN & $\mathrm{D}$ & $\mathrm{HD}$ \\
\hline 1 & W1 & W2 & NR & VL \\
\hline 2 & W1 & W2 & NSN & VL \\
\hline 3 & W1 & W2 & NSF & VL \\
\hline 4 & W1 & W2 & FA & LO \\
\hline 5 & W1 & NSW2 & NR & LO \\
\hline 6 & W1 & NSW2 & NSN & LO \\
\hline 7 & W1 & NSW2 & NSF & LO \\
\hline 8 & W1 & NSW2 & FA & $\mathrm{LH}$ \\
\hline 9 & W1 & N2 & NR & LH \\
\hline 10 & W1 & N2 & NSN & LH \\
\hline 11 & W1 & N2 & NSF & $\mathrm{HG}$ \\
\hline 12 & W1 & N2 & FA & $\mathrm{HG}$ \\
\hline 13 & W1 & $\mathrm{S} 2$ & NR & LH \\
\hline 14 & W1 & S2 & NSN & LH \\
\hline 15 & W1 & S2 & NSF & LH \\
\hline 16 & W1 & S2 & FA & $\mathrm{HG}$ \\
\hline 17 & NSW1 & W2 & NR & VL \\
\hline 18 & NSW1 & W2 & NSN & VL \\
\hline 19 & NSW1 & W2 & NSF & LO \\
\hline 20 & NSW1 & W2 & FA & LO \\
\hline 21 & NSW1 & NSW2 & NR & LO \\
\hline 22 & NSW1 & NSW2 & NSN & LO \\
\hline 23 & NSW1 & NSW2 & NSF & LH \\
\hline 24 & NSW1 & NSW2 & FA & LH \\
\hline 25 & NSW1 & N2 & NR & LH \\
\hline 26 & NSW1 & N2 & NSN & $\mathrm{LH}$ \\
\hline 27 & NSW1 & N2 & NSF & LH \\
\hline 28 & NSW1 & N2 & FA & $\mathrm{HG}$ \\
\hline 29 & NSW1 & $\mathrm{S} 2$ & NR & LH \\
\hline 30 & NSW1 & S2 & NSN & LH \\
\hline 31 & NSW1 & S2 & NSF & LH \\
\hline 32 & NSW1 & S2 & FA & HG \\
\hline 33 & N1 & W2 & NR & VL \\
\hline 34 & N1 & W2 & NSN & VL \\
\hline 35 & N1 & W2 & NSF & LO \\
\hline 36 & N1 & W2 & FA & LO \\
\hline 37 & N1 & NSW2 & NR & LO \\
\hline 38 & N1 & NSW2 & NSN & LO \\
\hline 39 & N1 & NSW2 & NSF & LH \\
\hline 40 & N1 & NSW2 & FA & LH \\
\hline 41 & N1 & N2 & NR & LH \\
\hline 42 & N1 & $\mathrm{N} 2$ & NSN & LH \\
\hline 43 & N1 & N2 & NSF & LH \\
\hline 44 & N1 & N2 & FA & $\mathrm{HG}$ \\
\hline 45 & N1 & S2 & NR & LH \\
\hline 46 & N1 & S2 & NSN & LH \\
\hline 47 & N1 & S2 & NSF & LH \\
\hline 48 & N1 & S2 & FA & HG \\
\hline 49 & $\mathrm{~S} 1$ & W2 & NR & VL \\
\hline 50 & $\mathrm{~S} 1$ & W2 & NSN & VL \\
\hline 51 & $\mathrm{~S} 1$ & W2 & NSF & VL \\
\hline 52 & $\mathrm{~S} 1$ & W2 & FA & VL \\
\hline 53 & $\mathrm{~S} 1$ & NSW2 & NR & VL \\
\hline 54 & $\mathrm{~S} 1$ & NSW2 & NSN & VL \\
\hline 55 & $\mathrm{~S} 1$ & NSW2 & NSF & VL \\
\hline 56 & $\mathrm{~S} 1$ & NSW2 & FA & LO \\
\hline 57 & $\mathrm{~S} 1$ & N2 & NR & LO \\
\hline 58 & $\mathrm{~S} 1$ & N2 & NSN & LO \\
\hline 59 & $\mathrm{~S} 1$ & N2 & NSF & LO \\
\hline 60 & $\mathrm{~S} 1$ & $\mathrm{~N} 2$ & FA & LH \\
\hline 61 & $\mathrm{~S} 1$ & $\mathrm{~S} 2$ & NR & LO \\
\hline 62 & $\mathrm{~S} 1$ & S2 & NSN & LO \\
\hline 63 & $\mathrm{~S} 1$ & S2 & NSF & LH \\
\hline 64 & $\mathrm{~S} 1$ & S2 & FA & LH \\
\hline
\end{tabular}
BS $(D M B)$ are kept the same. The output linguistic parameter is Handover Decision $(H D)$.

The FBHS2 operates as follows. First, after receiving the control information from MS, the POTLC check the quality of the signal. If the signal strength is still good enough the handover is not carried out. If the signal strength is lower than a predefined value, then based on CSSP, SSN and $D M B$, the FLC decides whether the handover is necessary or not. If the handover is not necessary the control is returned to the present BS, otherwise another check of the signal strength is done in PRTLC and the present signal strength is compared with the previous signal strength. When the present signal strength is lower than the previous signal, the handover procedure is carried out. 
Table 2. FRB2.

The term sets of CSSP, SSN and DMB are defined respectively as:

$$
\begin{aligned}
T(C S S P) & =\{\text { Small, Little Change, No Change, Big }\} \\
& =\{\text { SM,LC,NC,BG\}; } \\
T(S S N) & =\{\text { Weak, Not So Weak, Normal, Strong }\} \\
& =\{\text { WK, NSW, NO,ST }\} \\
T(D M B) & =\{\text { Near, Not So Near, Not So Far, Far }\} \\
& =\{\text { NR, NSN,NSF,FA }\} .
\end{aligned}
$$

The output linguistic parameter $T(H D)$ is defined as $\{$ Very Low, Low, Little High, High $\}=$ $\{V L, L O, L H, H G\}$.

The membership functions of FBHS2 are shown in Fig. 7 and the FRB2 is shown in Table 2.

\section{Simulation Results}

In both simulation systems, the cell shape is hexagonal and the coordinates of BSs are indicated as shown in Fig. 8. The BS is located in the center of the cell, the transmission antenna power is $10 \mathrm{~W}$, and cell radius is $2 \mathrm{~km}$. In Table 3 are shown the simulation parameters.

In Fig. 9 is showing the walking pattern for a MS. The MS moves in the cells: $(0,0) \rightarrow(2,-1) \rightarrow(0,0) \rightarrow(1,-2)$. In this case the MS is moving in the boundary of the cells, so the ping-pong effect happens. We evaluate FBHS1 and FBHS2 in the scenario of avoiding the ping-pong effect.

In Fig. 10 is shown the aggregated received power, while in Fig. 11, Fig. 12 and Fig. 13 are showing the received power from the $\mathrm{BS}(0,0), \mathrm{BS}(2,-1)$ ? Cand $\mathrm{BS}(1,-2)$. As can be seen from Fig. 11, when the MS is going far from the BS the received power is decreased, while when the MS
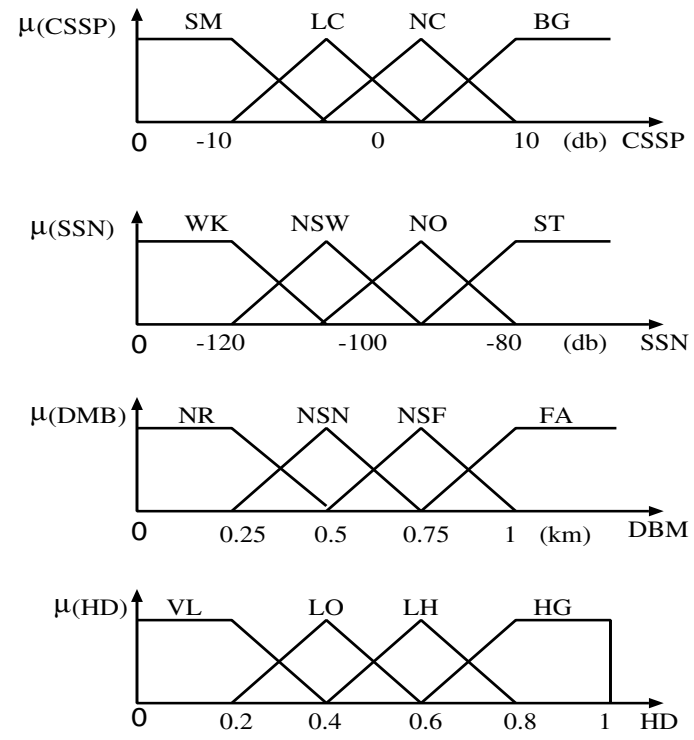

Figure 7. FBHS2 membership functions. 


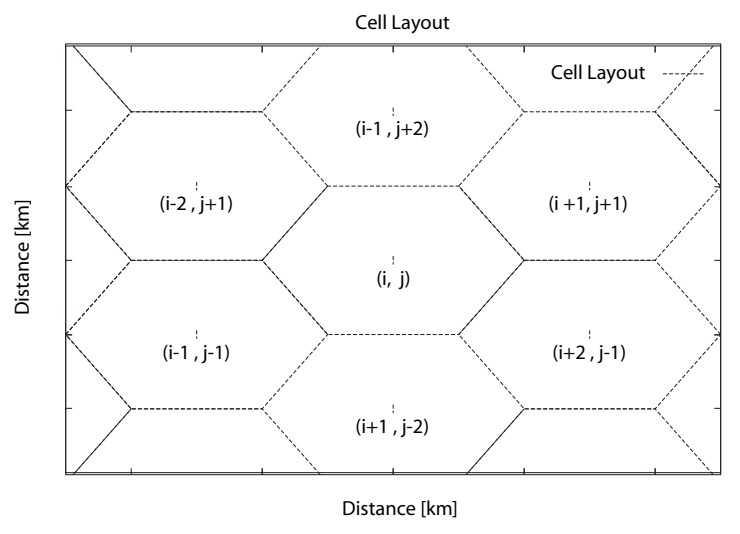

Figure 8. Cell layout.

Table 3. Simulation parameters.

\begin{tabular}{|c|c|}
\hline Distribution Law & Gaussian Distribution \\
\hline Number of Walks & $5 ? \mathrm{C} 10$ \\
\hline Random Types & $100 ? \mathrm{C} 200$ \\
\hline Cell Radius & $1 \mathrm{~km} ? \mathrm{C} 2 \mathrm{~km}$ \\
\hline Transmission Power & $10 \mathrm{~W} ? \mathrm{C} 20 \mathrm{~W}$ \\
\hline Frequency & $2000 \mathrm{MHz}$ \\
\hline Transmission Antenna Beam Tilting & $3^{\circ}$ \\
\hline Transmission Antenna Height & $40 \mathrm{~m}$ \\
\hline Receiving Antenna Height & $1.5 \mathrm{~m}$ \\
\hline Average Value for a Walk & $0.6 \mathrm{~km}$ \\
\hline$n$ & 1.1 \\
\hline
\end{tabular}

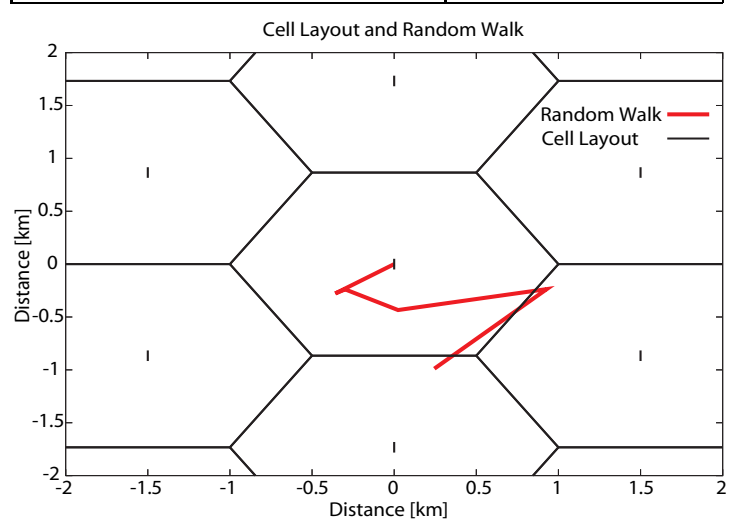

Figure 9. RW pattern.

culate the average values. The simulation results for FBHS1 and FHBS2 are shown in Table 4 and Table 5, respectively. As can be seen from Table 4, in most of the cases FBHS1 shows a good behavior. However, there are two values in the Measurement Point 3 that the value is more than 0.7. In this case, the FBHS1 carries out an un-necessary handover. As shown in Table 5, all the average values are smaller than 0.7 in FBHS2, therefore the FBHS2 system can avoid the ping-pong effect.

These results show that the selection of the parameters for making the handover decision is very important.

\section{Conclusions}

Many investigations have addressed handover algorithms for cellular communication systems. However, it

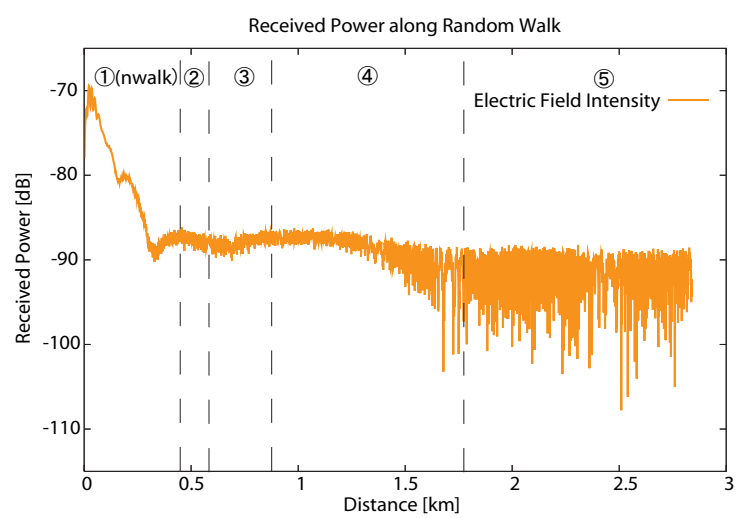

Figure 10. Aggregated received power.

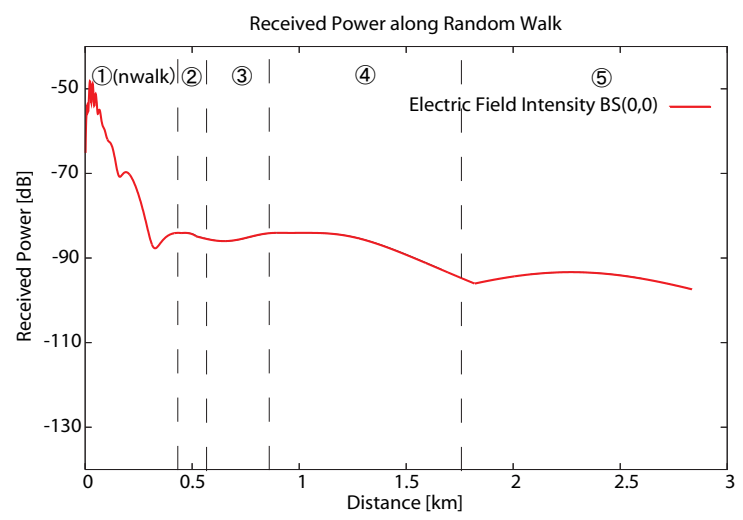

Figure 11. Received power from BS(0,0).

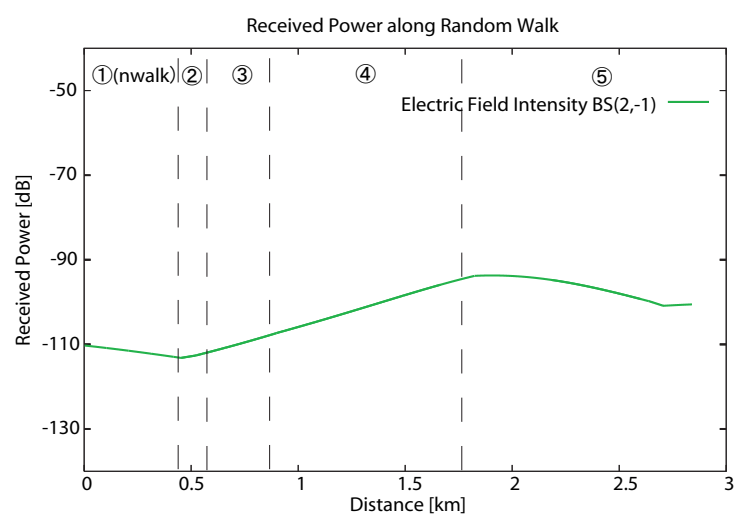

Figure 12. Received power from BS(2,-1).

is essentially complex to make handover decision considering multiple criteria. Sometimes, the trade-off of some criteria should be considered. Also, some of handover criteria information can be inherently imprecise, or the precise information is difficult to obtain. For this reason, we proposed two FL-based systems and evaluate their performance considering avoidance of the ping-pong effect, The simulation results show that the FBHS2 has a better behavior than FBHS1. 
Table 4. Simulation results for FBHS1.

\begin{tabular}{|c|c|c|c|c|c|c|}
\hline Measurement Points & \multicolumn{2}{|c|}{ Point 1} & \multicolumn{2}{|c|}{ Point 2} & \multicolumn{2}{|c|}{ Point 3} \\
\hline \multicolumn{7}{|c|}{$\overline{\overline{\text { Speed } 0 \mathrm{~km} / \mathrm{h}}}$} \\
\hline Present BS & -93.06 & -94.11 & -92.86 & -92.47 & -94.01 & -95.28 \\
\hline Neighbor BS & -93.36 & -92.49 & -92.77 & -93.98 & -93.99 & -91.28 \\
\hline Distance & 0.8804 & 0.9431 & 0.8684 & 0.8466 & 0.9367 & 1.0183 \\
\hline System Output Value & 0.595 & 0.629 & 0.602 & 0.576 & 0.623 & 0.704 \\
\hline \multicolumn{7}{|c|}{ Speed $10 \mathrm{~km} / \mathrm{h}$} \\
\hline Present BS & -95.06 & -96.11 & -94.86 & -94.47 & -96.01 & -97.28 \\
\hline Neighbor BS & -95.36 & -94.49 & -94.77 & -95.98 & -95.99 & -93.28 \\
\hline Distance & 0.8858 & 0.9431 & 0.8684 & 0.8466 & 0.9367 & 1.0183 \\
\hline System Output Value & 0.598 & 0.649 & 0.600 & 0.578 & 0.623 & 0.708 \\
\hline \multicolumn{7}{|c|}{ Speed $20 \mathrm{~km} / \mathrm{h}$} \\
\hline Present BS & -97.06 & -98.11 & -96.86 & -96.47 & -98.01 & -99.28 \\
\hline Neighbor BS & -97.36 & -96.49 & -96.77 & -97.98 & -97.99 & -95.28 \\
\hline Distance & 0.8804 & 0.9431 & 0.8684 & 0.8466 & 0.9367 & 1.0183 \\
\hline System Output Value & 0.568 & 0.621 & 0.572 & 0.538 & 0.590 & 0.696 \\
\hline \multicolumn{7}{|c|}{ Speed $30 \mathrm{~km} / \mathrm{h}$} \\
\hline Present BS & -99.06 & -100.11 & -98.86 & -98.47 & -100.01 & -101.28 \\
\hline Neighbor BS & -99.36 & -98.49 & -98.77 & -99.98 & -99.99 & -97.28 \\
\hline Distance & 0.8804 & 0.9431 & 0.8684 & 0.8466 & 0.9367 & 1.0183 \\
\hline System Output Value & 0.522 & 0.585 & 0.531 & 0.482 & 0.542 & 0.662 \\
\hline \multicolumn{7}{|c|}{ Speed $40 \mathrm{~km} / \mathrm{h}$} \\
\hline Present BS & -101.06 & -102.11 & -100.86 & -100.47 & -102.01 & -103.28 \\
\hline Neighbor BS & -101.36 & -100.49 & -100.77 & -101.98 & -101.99 & -99.28 \\
\hline Distance & 0.8804 & 0.9431 & 0.8684 & 0.8466 & 0.9367 & 1.0183 \\
\hline System Output Value & 0.534 & 0.597 & 0.521 & 0.497 & 0.590 & 0.672 \\
\hline \multicolumn{7}{|c|}{ Speed $50 \mathrm{~km} / \mathrm{h}$} \\
\hline Present BS & -103.06 & -104.11 & -101.86 & -104.47 & -104.01 & -105.28 \\
\hline Neighbor BS & -103.36 & -102.49 & -102.77 & -103.98 & -103.99 & -101.28 \\
\hline Distance & 0.8804 & 0.9431 & 0.8684 & 0.8466 & 0.9367 & 1.0183 \\
\hline System Output Value & 0.576 & 0.625 & 0.566 & 0.549 & 0.600 & 0.668 \\
\hline
\end{tabular}

Table 5. Simulation results for FBHS2.

\begin{tabular}{|c|c|c|c|c|c|c|}
\hline Measurement Points & \multicolumn{2}{|c|}{ Point 1} & \multicolumn{2}{|c|}{ Point 2} & \multicolumn{2}{|c|}{ Point 3} \\
\hline \multicolumn{7}{|c|}{$\overline{\text { Speed } 0 \mathrm{~km} / \mathrm{h}}$} \\
\hline CSSP BS & -2.710 & -3.697 & -1.289 & 0.3877 & -1.189 & -1.270 \\
\hline Neighbor BS & -93.36 & -92.49 & -92.77 & -92.77 & -94.01 & -95.28 \\
\hline Distance & 0.8858 & 0.9453 & 0.8684 & 0.8466 & 0.9367 & 1.0183 \\
\hline System Output Value & 0.693 & 0.600 & 0.539 & 0.497 & 0.571 & 0.600 \\
\hline \multicolumn{7}{|c|}{ Speed $10 \mathrm{~km} / \mathrm{h}$} \\
\hline CSSP BS & -2.710 & -3.697 & -1.289 & 0.3877 & -1.189 & -1.270 \\
\hline Neighbor BS & -95.36 & -94.49 & -94.77 & -94.77 & -96.01 & -97.28 \\
\hline Distance & 0.8858 & 0.9427 & 0.8684 & 0.8466 & 0.9367 & 1.0183 \\
\hline System Output Value & 0.693 & 0.600 & 0.583 & 0.542 & 0.600 & 0.618 \\
\hline \multicolumn{7}{|c|}{ Speed $20 \mathrm{~km} / \mathrm{h}$} \\
\hline CSSP BS & -2.710 & -3.697 & -1.289 & 0.3877 & -1.189 & -1.270 \\
\hline Neighbor BS & -97.36 & -96.49 & -96.77 & -96.77 & -98.01 & -99.28 \\
\hline Distance & 0.8858 & 0.9401 & 0.8684 & 0.8466 & 0.9367 & 1.0183 \\
\hline System Output Value & 0.693 & 0.600 & 0.614 & 0.574 & 0.624 & 0.640 \\
\hline \multicolumn{7}{|c|}{ Speed $30 \mathrm{~km} / \mathrm{h}$} \\
\hline CSSP BS & -2.710 & -3.697 & -1.289 & 0.3877 & -1.189 & -1.270 \\
\hline Neighbor BS & -99.36 & -98.49 & -98.77 & -98.77 & -100.0 & -101.3 \\
\hline Distance & 0.8858 & 0.9376 & 0.8684 & 0.8466 & 0.9367 & 1.0183 \\
\hline System Output Value & 0.693 & 0.600 & 0.632 & 0.584 & 0.645 & 0.657 \\
\hline \multicolumn{7}{|c|}{ Speed $40 \mathrm{~km} / \mathrm{h}$} \\
\hline CSSP BS & -2.710 & -3.697 & -1.289 & 0.3877 & -1.189 & -1.270 \\
\hline Neighbor BS & -101.4 & -100.5 & -100.8 & -100.8 & -102.0 & -103.3 \\
\hline Distance & 0.8858 & 0.9351 & 0.8684 & 0.8466 & 0.9367 & 1.0183 \\
\hline System Output Value & 0.693 & 0.600 & 0.631 & 0.582 & 0.656 & 0.662 \\
\hline \multicolumn{7}{|c|}{ Speed $50 \mathrm{~km} / \mathrm{h}$} \\
\hline CSSP BS & -2.710 & -3.697 & -1.289 & 0.3877 & -1.189 & -1.270 \\
\hline Neighbor BS & $\begin{array}{l}-103.4 \\
\end{array}$ & -102.5 & -102.8 & -102.8 & -104.0 & -105.3 \\
\hline Distance & 0.8858 & 0.9327 & 0.8684 & 0.8466 & 0.9367 & 1.0183 \\
\hline System Output Value & 0.693 & 0.600 & 0.631 & 0.582 & 0.656 & 0.663 \\
\hline
\end{tabular}




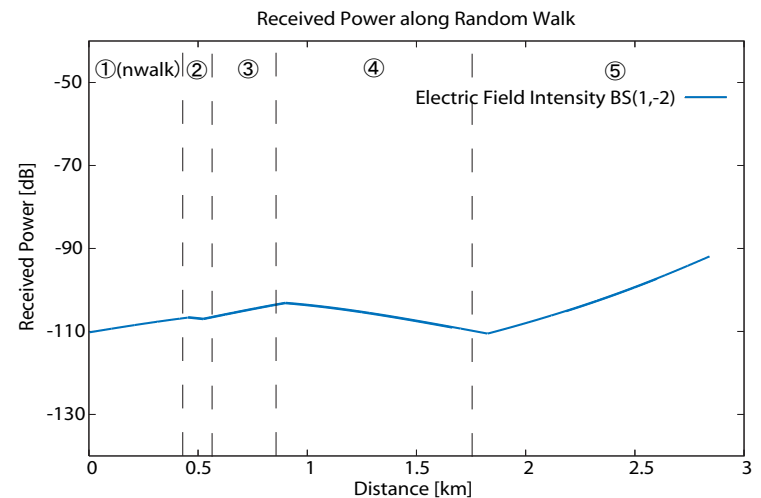

Figure 13. Received power from BS(1,-2).

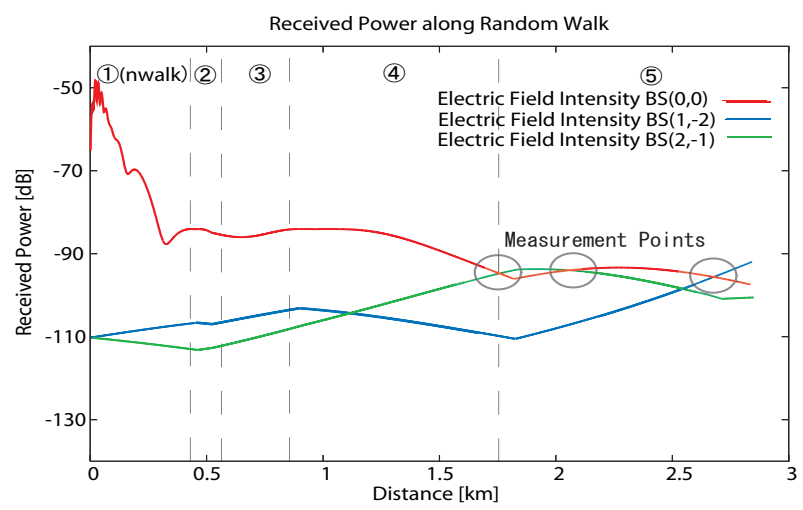

Figure 14. 3 measurement points.

In the future, we would like to compare the performance of the proposed system with other non-fuzzy-based handover algorithms.

\section{References}

[1] R. Berezdivin, R. Breining, R. Topp, "NextGeneration Wireless Communication Concepts and Technologies", IEEE Communication Magazine, Vol. 40, No. 3, pp. 108-116, 2002.

[2] Y. Guo, H. Chaskar, "Class-Based Quality of Service over Air Interfaces in 4G Mobile Networks", IEEE Communication Magazine, Vol. 40, No. 3, pp. 132137, 2002.

[3] W. Wang, X. Wang, A.A. Nilsson, ”Energy-Efficient Bandwidth Allocation in Wireless Networks: Algorithms, Analysis, and Simulations", IEEE Transactions on Wireless Communications, Vol. 5, No. 5, pp. 1103-1114, 2006.

[4] Y. Fang, Y. Zhang, "Call Admission Control Schemes and Performance Analysis in Wireless Mobile Networks", IEEE Transactions on Vehicular Technology, Vol. 51, No. 2, pp. 371-382, 2002.

[5] K. D. Wong, D.C. Cox, "A Pattern Recognition System for Handoff Algorithms", IEEE J-SAC, Vol. 18, No. 7, pp. 1301-1312, 2000.
[6] S. Kovvuri, V. Pandey, D. Ghosal, B. Mukherjee, D. Sarkar, "A Call-Admission Control (CAC) Algorithm for Providing Guaranteed QoS in Cellular Networks", International Journal of Wireless Information Networks, Vol. 10, No. 2, pp. 73-85, 2003.

[7] H.P. Lin, R.T. Juang, D.B. Lin, "Validation of an Improved Location-Based Handover Algorithm Using GSM Measurement Data", IEEE Transactions on Mobile Computing, Vol. 4, No. 5, pp. 530-536, 2005.

[8] P. Fiengo, G. Giambene, E. Trentin, "Neural-based Downlink Scheduling Algorithm for Broadband Wireless Networks", Computer Communication, Vol. 30, No. 2, pp. 207-218, 2007.

[9] L. Barolli, A. Koyama, T. Suganuma, N. Shiratori, "GAMAN: A GA Based QoS Routing Method for Mobile Ad-hoc Networks", Journal of Interconnection Networks (JOIN), Vol. 4, No. 3, pp. 251-270, 2003.

[10] T. Onel, C. Ersoy, E. Cayirci, "A Fuzzy Inference System for the Handoff Decision Algorithms in the Virtual Cell Layout Base Tactical Communications System”, IEEE Military Communications Conference (MILCOM-2002), Vol. 1, pp. 436-441, 2002.

[11] P. M. L. Chan, R. E. Sheriff, Y.F. Hu, P. Conforto, C. Tocci, "Mobility Management Incorporating Fuzzy Logic for a Heterogeneous IP Environment", IEEE Communications Magazine, Vol. 39, No. 12, pp. 4251, 2001.

[12] L. Barolli, A. Durresi, F. Xhafa, A. Koyama, "A Fuzzy Based Handover System for Wireless Cellular Networks: A Case Study for Handover Enforcement", Proc. of NBiS-2008, Torino, Italy, pp. 212-222, September 2008.

[13] L. Barolli, J. Anno, F. Xhafa, A. Durresi, A. Koyama, "A Context-Aware Fuzzy-Based Handover System for Wireless Cellular Networks and Its Performance Evaluation", Journal of Mobile Multimedia (JMM), Vol. 4. No. 3/4, pp. 241-258, October 2008.

[14] S. Mohanty, I.F. Akyildiz, "A Cross-Layer (Layer 2+3) Handoff Management Protocol for NextGeneration Wireless Systems", IEEE Transactions on Mobile Computing, Vol. 5, No. 10, pp. 1347-1360, 2006.

[15] F. Yu, Y. Krishamurthy, "Optimal Joint Session Admission Control in Integrated WLAN and CDMA Cellular Networks with Vertical Handoff", IEEE Transactions on Mobile Computing, Vol. 6, No. 1, pp. 126139, 2007.

[16] D. Dubois, H. Prade, R. Yager, (Eds.), "Fuzzy Sets for Intelligent Systems", Morgan Kaufman Publishers, 1993. 